\title{
Performance Analysis of IEEE 802.11n MAC Enhancements over Block Fading Channels
}

\author{
Roger Pierre Fabris Hoefel
}

\begin{abstract}
Assuming a Nakagami-m block fading channel, we derive a theoretical medium access control (MAC) and physical (PHY) cross-layer model to estimate the saturation net throughput of IEEE 802.11 wireless local area networks (WLANs) with MAC enhancements. It is analytically shown that these MAC improvements, that consist of modifications of the plain IEEE 802.11 distribution coordination function (DCF) MAC protocol in order to support concatenation and multiframe transmission techniques, allow a substantial improvement of the net throughput.
\end{abstract}

Keywords: WLANs, 802.11n, 802.11a, cross-layer, PHY, MAC.

\section{INTRODUCTION}

IEEE 802.11 task group N (TGn) goals are to achieve 100 Mbps net throughput, after subtracting all the overhead for protocol management tasks, like preambles, inter-frame spacing (IFS), control and management frames and so forth. It is well-known that the IEEE 802.11 DCF MAC protocol has several overheads that limit the maximum throughput [1]. Therefore, different from PHY layer enhancements of $802.11 \mathrm{a} / .11 \mathrm{~g}$ family of specifications, the upcoming IEEE $802.11 \mathrm{n}$ standard will provide higher throughput with both MAC and PHY layer improvements. In next paragraph, we describe some research activities that have been focusing on MAC layer enhancement issues.

Y. Xiao in [2-3] proposed several MAC enhancements based on packing, concatenation and multiframe transmission techniques. Liu and A. Stephens presented in [4] an analytical model to estimate and optimize the capacity of infrastructure WLANs that support bidirectional MAC frame aggregation. L. X. Cai et. al. derived in [5] an analytical model to investigate the performance of enhanced MAC mechanisms for the IEEE 802.11n standard, where they focused on MAC improvements to reduce the access point (AP) bottleneck effect in infrastructure WLANs. It was proposed in [6] a centralized MAC protocol that uses time division multiple access (TDMA) to divide all the stations (STAs) in few disjoint groups to avoid all STAs transmitting their frames simultaneously.

The main contribution of this paper consist of an analytical first order MAC and PHY cross layer model that allows estimating the saturation goodput (the saturation net throughput) of IEEE DCF 802.11 MAC enhancements schemes over Nakagami-m block fading channels. We shall analyze some of the MAC protocols enhancements originally proposed and simulated by Y. Xiao in [2-3]. The present theoretical model is designated as MAC and PHY cross-layer because multiple access protocols, traffic load,

Manuscript received March, 17, 2008; revised June, 22, 2008.

Roger Pierre Fabris Hoefel is with Department of Electrical Engineering, Federal University of Rio Grande do Sul (UFRGS), Porto Alegre, Rio Grande do Sul, Brazil; E-mail: roger.hoefel@ufrgs.br.

This work was partially funded by CNPq, project \#474441/2007-5 multipath fading channel, modulation/coding schemes and receiver structures are jointly taken into account to estimate the goodput.

To accomplish our goals, this paper is organized as follows. Section II summarizes an analytical model that we have been using to estimate the goodput of the IEEE DCF 802.11 MAC protocol. Section III presents expressions that we have been developing to estimate the frame success probability over block fading (i.e. correlated over frequency and time) fading channels. The previous two sections consist of a fundamental enabling background that allows us to develop in the following section the original contributions of this paper. Section IV derives an analytical model to estimate the net throughput of two 802.11 DCF MAC enhancement protocols. Section V uses analytical and simulation tools to compare the performance of the MAC protocols modeled in the previous sections. Finally, Section VI presents our conclusions and future research activities.

\section{IEEE 802.11 CONVENTIONAL DCF MAC PROTOCOL}

The mathematical details of the results presented in this section may be found in [7]. We shall use the IEEE 802.11a PHY layer since our main concern in this paper is to carry out a theoretical comparative analyses of IEEE 802.11 DCF MAC protocols enhancements.

Fig. 1 shows the time diagram for the atomic transmission cycle used by the IEEE 802.11 DCF requestto-send/clear-to-send (RTS/CTS) clearing technique [8]. DIFS stands for DCF inter-frame spacing (IFS), PIFS for point coordination IFS, SIFS for short IFS, ACK for acknowledgment (ACK) control frame and NAV for network allocation vector. The mandatory DCF is founded on the Carrier Sense Multiple Access with Collision Avoidance (CSMA/CA) contention protocol, where physical carrier sensing and virtual carrier-sensing functions are implemented to manage this process [8].

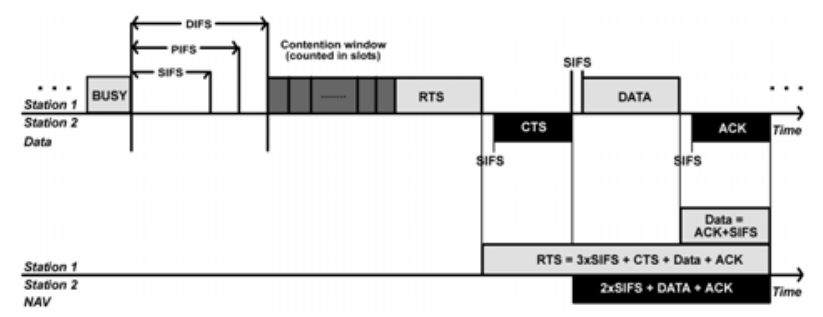

Fig. 1. The atomic cycle for the successful transmission using RTS/CTS.

Assuming saturation traffic (i.e. all the STAs always have data to transmit [9]), the conditional probability that a transmitted PHY layer protocol data unit (PPDU) has done a collision given that a source STA transmits in a slot time of length $\sigma$ can be estimated by 


$$
p=1-(1-\tau)^{n-1},
$$

where $n$ is the number of STAs in the system.

The probability that a STA transmits in a randomly chosen slot time can be estimated by

$$
\tau=\frac{b_{0,0}}{S \cdot(1-p)}=\frac{b_{0,0}}{S_{r t s} \cdot S_{c t s} \cdot S_{d} \cdot S_{a c k} \cdot(1-p)},
$$

where the probability that an atomic transmission cycle is successful is given by

$$
S=S_{r t s} \cdot S_{c t s} \cdot S_{d} \cdot S_{a c k},
$$

and

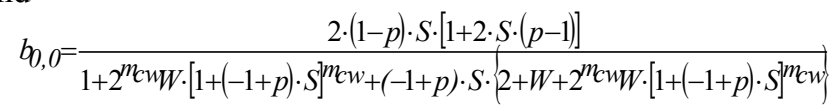

It is used the following notation:

1. $b_{0,0}$ is the probability that a STA is at the slot time 0 of the $0^{\text {th }}$ backoff stage.

2. $S_{c t s,} S_{r t s}$ and $S_{a c k}$ denote the probability that the RTS, CTS and ACK control frames be transmitted with success;

3. $S_{d}$ denotes the probability that the transmission of an MPDU frame is successful;

4. The minimum $\mathrm{CW}$ is labeled as $W$.

5. The window size at the backoff stage $i$ is given by $\mathrm{W}_{\mathrm{i}}=2^{\mathrm{i}} \mathrm{W}$, where $i \in\left(0, m_{c w}\right)$.

6. The number of backoff stages is $m_{c w}$.

The nonlinear system given by (1) and (2) is used to estimate $p$ and $\tau$.

\section{Frame Success Probability OVER BLOCK FAdING CHANNELS}

It is assumed that the errors inside of the hard decision Viterbi convolutional forward error correcting code (FEC) decoder are interdependent. Pursley and Taipale have shown that the upper bound for a successful transmission of a frame with $l$ octets is given by [10]

$$
S\left(l, \gamma_{b}, m\right)<\left[1-P_{e}\left(\gamma_{b}, m\right)\right]^{8 l},
$$

where $P_{e}\left(\gamma_{b}, m\right)$ is the union bound on the probability of decoding error. $\gamma_{b}$ is the signal-to-interference-plus-noiseratio (SINR) per bit and $m$ denotes the PHY mode $m$.

For a block-fading channel, this upper bound can be modified to [7]

$$
S\left(l, \gamma_{b}, m\right)<\int_{\gamma_{\mathrm{inf}}}^{\infty}\left[1-\operatorname{Pe}\left(\gamma_{b}, m\right)\right]^{8 l} p\left(\gamma_{b}\right) d \gamma_{b},
$$

where the lower limit of this definite integral is chosen so that the inequality (7) is satisfied.

$$
\left[1-P e\left(\gamma_{b}, m\right)\right]^{8 l} \leq 1 \text { for } \gamma_{b} \geq \gamma_{\text {inf }}
$$

Considering a Nakagami-m fading channel; a maximum ratio combining (MRC) receiver matched with the channel diversity; and that the same average power $\Omega$ is received at each diversity branch, then the probability distribution function (pdf) of the SINR per bit at the Viterbi decoder input is of gamma kind [11], i.e.

$$
p\left(\gamma_{b}\right)=\frac{1}{\Gamma\left(L m_{n}\right)}\left(\frac{m_{n}}{\bar{\gamma}_{b}}\right)^{L m_{n}}\left(\gamma_{b}\right)^{L m_{n}-1} \exp \left(-\frac{m_{n} \gamma_{b}}{\bar{\gamma}_{b}}\right) \text { if } \gamma_{b}>0, m_{n} \geq 0.5,(8)
$$

where $\Gamma($.$) is the gamma function, m_{n}$ is the Nakagami-m fading figure ( $m_{n}=1$ for Rayleigh fading), $\bar{\gamma}_{b}$ is the average
SINR per bit at the Viterbi decoder input and $L$ is the number of receiver diversity branches.

In a block fading channel, the fading changes slowly in each atomic transmission cycle (see Fig. 1) and it is assumed uncorrelated in different transmission cycles. Therefore, in systems like IEEE 802.11 we must consider two basic cases: (1) all frames use the same modulation scheme; (2) the modulation scheme used by the control frames (i.e. RTS, CTS and ACK) is different from that one used in the transmission of MAC PDU (MPDU) frames. For WLANS that use 802.11a/.11g PHY layers, the typical cases 1 and 2 can be exemplified with PHY modes 5 and 8, respectively.

Hereafter, we will use the following notation: $N_{r t s}, N_{c t s}$, $N_{d}$ and $N_{a c k}$ are the total number of octets (i.e. preamble, headers and information fields) of the PHY layer PDU (PPDU) that transports the RTS, CTS, MPDU and ACK frames, respectively. A comprehensive detailed description of these terms may be found in [8].

\section{A. PHY Mode 5 (16QAM@24Mbps)}

The mode 5 of IEEE 802.11a PHY layer has the following main characteristics: (a) the gross bit rate is of $24 \mathrm{Mbps}$; (b) it uses the 16 rectangular quadrature amplitude modulation (16QAM) scheme; (c) the FEC scheme

uses a convolutional code with generator polynomials, $\boldsymbol{g}_{0}=(133)_{8}$ and $\boldsymbol{g}_{1}=(171)_{8}$, of rate $R_{c}=1 / 2$ and constraint length $K=7$

When a MPDU is transmitted using the PHY mode 5, then all the control and data frames are transmitted using the PHY mode 5. Hence, the $S_{r t s}$ and $S_{c t s}$ can be estimated by

$$
\begin{aligned}
& S_{r t s}(m)=S\left(N_{r t s}, \gamma_{b}, m_{r t s}\right) . \\
& S_{c t s}(m)=P\{C T S \text { is correct } / R T S \text { was correct }\} ; \\
& S_{c t s}(m)=\frac{S\left(N_{r t s}+N_{c t s}, \gamma_{b}, m_{c t s}\right)}{S_{r t s}} .
\end{aligned}
$$

The probability that $n_{f}$ MPDUS are transmitted with success given that RTS and CTS control frames were successfully transmitted can be approximated by

$$
S_{d}(m)=P\left\{n_{f} M P D U \text { are correct } / R T S \text { and CTS were correct }\right\} \text {; }
$$

$$
S_{d}\left(m, n_{f}\right)=\frac{S\left(N_{r t s}+N_{c t s}+n_{f} \cdot N_{d, \gamma_{b}, m_{d}}\right)}{S_{r t s} S_{c t s}} .
$$

Finally, the frame success probability for the ACK control frame can be estimated by

$S_{a c k}\left(m, n_{f}\right)=P\{A C K$ is correct $/ R T S, C T S$ and $n f$ MPDU werecorrect $\}$

$S_{a c k}\left(m, n_{f}\right)=\frac{S\left(N_{r t s}+N_{c t s}+n_{f} N_{d}+N_{a c k}, \gamma_{b}, m_{a c k}\right)}{S_{r t s} S_{c t s} S_{d}}$.

The frame success probability can be estimated by (5), where the union bound on the probability of decoding error is given by

$$
P_{e}\left(\gamma_{b}, m\right)<11 P_{10}\left(\gamma_{b}, m\right)+38 P_{12}\left(\gamma_{b}, m\right)+193 P_{14}\left(\gamma_{b}, m\right)+\cdots,
$$

Assuming that the convolutional FEC is decoded using hard-decision Viterbi decoding, then (16) and (17) model the probability of incorrectly selecting a path when the Hamming distance $d$ is even and odd, respectively.

$$
P_{d}\left(\gamma_{b}, m\right)=\frac{1}{2}\left(\begin{array}{l}
d \\
d / 2
\end{array}\right) p_{m}^{d / 2}\left(1-\rho_{m}\right)^{d / 2}+\sum_{k=d / 2+1}^{d}\left(\begin{array}{l}
d \\
k
\end{array}\right) \rho_{m}^{k}\left(1-\rho_{m}\right)^{d-k} .
$$




$$
P_{d}\left(\gamma_{b}, m\right)=\sum_{k=(d+1) / 2}^{d}\left(\begin{array}{l}
d \\
k
\end{array}\right) \rho_{m}^{k}\left(1-\rho_{m}\right)^{d-k} .
$$

The average bit error rate (BER) when the PHY mode $m$ uses QAM modulation scheme is given by [12]

$$
\begin{aligned}
\rho_{m}= & \frac{\sqrt{M}-1}{\sqrt{M} \log _{2} \sqrt{M}} \operatorname{erfc}\left(\sqrt{\frac{2 \log _{2} M \cdot \gamma_{b} \cdot R_{c}}{2(M-1)}}\right) \\
& +\frac{\sqrt{M}-2}{\sqrt{M} \log _{2} \sqrt{M}} \operatorname{erfc}\left(\sqrt{\frac{3 \log _{2} M \cdot \gamma_{b} \cdot R_{c}}{2(M-1)}}\right),
\end{aligned}
$$

where $M=16$ and $R_{c}=1 / 2$ por PHY mode 5 .

\section{B. PHY Mode 8(64QAM@54Mbps)}

The mode 8 of IEEE 802.11a PHY layer has following main characteristics : (a) the gross bit rate is of $54 \mathrm{Mbps}$; (b) it uses the 64 rectangular QAM 64QAM scheme; (c) the FEC uses a convolutional code with generator polynomials with rate $\mathrm{R}_{\mathrm{c}}=3 / 4$, which are obtained by puncturing the original rate- $1 / 2$ code used by PHY mode 5 . The union bound on the probability of decoding error of this punctured convolutional code can be upper bounded by

$$
P_{e}\left(\gamma_{b}, m\right)<8 P_{5}\left(\gamma_{b}, m\right)+31 P_{6}\left(\gamma_{b}, m\right)+160 P_{7}\left(\gamma_{b}, m\right)+\cdots
$$

When the MPDU is transmitted using the PHY mode 8 , then the control frames are transmitted using the PHY mode 5. Thus, the $S_{r t s}$ and $S_{c t s}$ are given by (9) and (11) with $m=5$.

The probability that $n_{f}$ MPDUs are transmitted successfully can be approximatted by

$P\left\{n_{f}\right.$ MPDUs are correct/ RTS and CTS wereTX correctly $\}=$

$$
=\frac{P\left\{n_{f} M P D U s, R T S, C T S\right\}}{P\{R T S, C T S\}} \approx \frac{P\left\{n_{f} M P D U s\right\}}{P\{R T S, C T S\}}(20)
$$

since $N_{m p}>>\left(N_{r t s}+N_{c t s}\right)$, and the MPDU is transmitted using the $P H Y$ mode 8 (i.e. a signaling scheme with lesser immunity to noise and interference than PHY mode 5 used to transmit the control frames). Therefore,

$$
S_{d}\left(m_{d}, n_{f}\right) \cong \frac{S\left(n_{f} N_{\left.d, \gamma_{b}, m_{d}\right)}\right.}{S_{r t s}(5) S_{c t s}(5)}=\frac{S\left(n_{f} N_{d,} \gamma_{b}, m_{d}\right)}{S\left(N_{r t s}+N_{c t s}, \gamma_{b}, 5\right)},
$$

where $m_{d}=8$.

The ACK control frame is transmitted using the $P H Y$ mode 5, while the MPDU is transmitted using the $P H Y$ mode 8 (i.e. a signaling scheme more suitable to the decoding errors). Thus obviously the ACK control frame success probability can be approximated by (22) for block fading channels.

$$
S_{a c k}(m)=P\{A C K \text { is ack } / R T S, \text { CTS and MPDU were ack }) \cong 1 .
$$

\section{IEEE 802.11n MAC ENHANCEMENTS}

A qualitative description of IEEE 802.11 DCF MAC protocols enhancements can be found in [2]. In this section, we analyze theoretically the performance of multiframe transmission and concatenation MAC enhancements schemes operating over block fading channels.

The atomic cycle for the MAC protocols analyzed in this paper are depicted at Fig. 2. The time diagrams do not show the random access that occurs in the CW and the RTS/CTS clearing technique to avoid overcrowded details in Fig. 2. However, we notice that our analytical model takes into account the contention effects and the transmission of RTS and CTS control frames, as detailed shown in Fig. 1, before the transmission of the Frame 1.

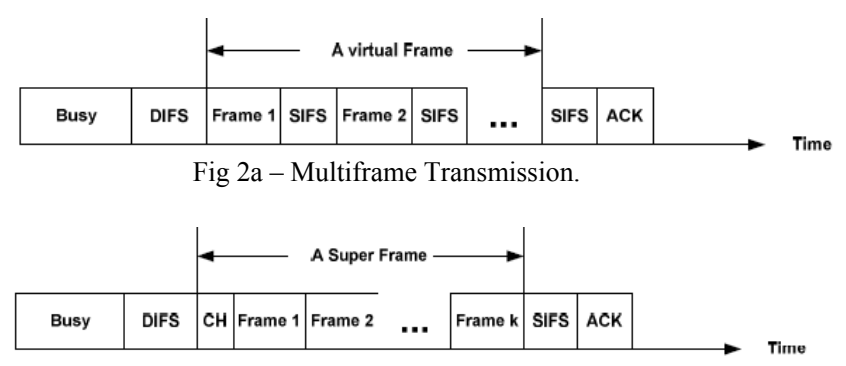

Fig 2b-Concatenation, $\mathrm{CH}$ : concatenation header [2].

Fig 2- Simplified cycle atomic for 802.11 MAC enhancements.

\section{A. MULTIPLE FRAME TRANSMISSION (MFT)}

Fig. 2a depicts the MFT scheme. Given that the channel was successfully reserved by the RTS/CTS handshake procedure, then the source STA transmits $n_{f}$ frames. The transmission is not aborted if an MPDU frame is corrupted due to noise and interference. The ACK control frame is transmitted by the target STA if any one MPDU is transmitted with success. This ACK control frame has a field to indicate which MPDUs were correctly received. Hence, if the MPDUS transmissions were independent, then the average number of MPDU frames transmitted in the atomic transmission cycle time would be given by

$$
\overline{N_{f}}=\sum_{k=1}^{n_{f}}\left(\begin{array}{l}
n_{f} \\
k
\end{array}\right) \cdot k \cdot\left(S_{d}\right)^{k} \cdot\left(1-S_{d}\right)^{n_{f}-k} .
$$

However, as we have assumed a block fading channel, then we can use the following estimation

$$
\overline{N_{f}} \approx n_{f} \cdot S_{d}(m, l),
$$

i.e. if one MPDU frame is transmitted with success, then all MPDUS are transmitted successfully due to the block fading assumption.

The goodput can be estimated by

$$
G_{b p s}(m)=\frac{8 \cdot l_{p l} \overline{N_{f}}}{\bar{T}} \cdot\left(P_{s} \cdot P_{t r}\right) \cdot\left(S_{r t s} \cdot S_{c t s}\right) \cdot\left(S_{a c k}\right),
$$

where $l_{p l}$ is the number of payload octets of the MPDU frame. Notice that the MPDU success probability is explicitly included in the estimation of $\overline{N_{f}}$.

The probability that there is no collision on the channel conditioned to the fact that at least one STA transmits is given by

$$
P_{S}=\frac{n \cdot \tau \cdot(1-\tau)^{n-1}}{P_{t r}}=\frac{n \cdot \tau \cdot(1-\tau)^{n-1}}{1-(1-\tau)^{n}},
$$

where $P_{t r}$ is the probability that at least one transmission occurred in the considered slot time of length $\sigma$.

The average cycle time consists of sum of eight terms:

$$
\bar{T}=\bar{B}_{s}+\bar{B}_{f 1}+\bar{B}_{f 2}+\bar{B}_{f 3}+B_{f 4}+B_{f 5}+B_{f 6}+\bar{I}
$$

In the following, we shall define and model the terms of the above equation.

The average time in which a slot time is idle is given by

$$
\bar{I}=\left(1-P_{t r}\right) \cdot \sigma \text {. }
$$

The average busy time for a successful transmission 
cycle must take into account that on average $\overline{N_{f}}$ MPDUs are transmitted with success on each transmission cycle and that an ACK control frame is transmitted if at least one MPDU is successfully received. Therefore,

$$
\begin{aligned}
\bar{B}_{S}= & P_{s} \cdot P_{t r} \cdot S_{r t s} \cdot S_{c t s} \cdot \\
& \left\{\left[D I F S+T_{r t s}\left(m_{r t s}\right)+a+S I F S+T_{c t s}\left(m_{c t s}\right)+a+S I F S\right]+\right. \\
& \left.\overline{N_{f}} \cdot\left(T_{d}\left(m_{d}\right)+S I F S+a\right)+S_{d} S_{a c k}\left[T_{a c k}\left(m_{a c k}\right)+a\right]\right\}
\end{aligned}
$$

where $a$ is the propagation delay, $T_{r t s}\left(m_{r t s}\right)$ is the time necessary to transmit the RTS control frame when it is used the PHY mode $m_{r t s}$. Correspondingly, $T_{c t s}\left(m_{c t s}\right), T_{d}\left(m_{d}\right)$ and $T_{a c k}\left(m_{a c k}\right)$ denote the time necessary to transmit CTS, MPDU and ACK frames when the PHY modes $m_{c t s}, m_{d}$ and $m_{a c k}$ are used, respectively.

Assuming the IEEE 802.11a PHY layer, then the time period spent to transmit a MPDU with a payload of $l_{p l}$ octets when it is used the PHY mode $m_{d}$ is given by

$$
\begin{aligned}
T_{d}\left(m_{d}\right)= & t P C L P \text { Preamble }+t P C L P \_S I G+ \\
& {\left[\frac{l_{p l}+34+(16+6) / 8}{B p S(m)}\right] t \text { Symbol } . }
\end{aligned}
$$

Some IEEE 802.11a parameters are: (a) $t$ Symbol $=4 \mu$; (b) the PHY layer convergence procedure (PLCP) preamble duration, $t P C L P$ Preamble, is equal to $12 \mu \mathrm{s}$; (c) the PCLP field duration, $t P C L P \_S I G$, is equal to $4 \mu \mathrm{s}$.

Expressions to calculate the length of RTS, CTS, and ACK frames may be found in [7].

The following time periods model the average waste time that the channel is busy with unsuccessful frame transmissions:

1. $\bar{B}_{f 1}$ : collision in the transmission of RTS control frame.

$$
\bar{B}_{f 1}=P_{t r} \cdot\left(1-P_{s}\right) \cdot\left[D I F S+T_{r t s}\left(m_{r t s}\right)+a\right] .
$$

2. $\bar{B}_{f 2}:$ no-collision + (RTS corruption).

$$
\bar{B}_{f 2}=P_{t r} \cdot P_{s} \cdot\left(1-S_{r t s}\right)\left[D I F S+T_{r t s}\left(m_{r t s}\right)+a\right] .
$$

3. $\bar{B}_{f 3}$ : no-collision $+R T S+(C T S$ corruption $)$.

$$
\bar{B}_{f 3}=P_{t r} \cdot P_{s} \cdot S_{r t s} \cdot\left(1-S_{c t s}\right)\left[\begin{array}{l}
D I F S+T_{r t s}\left(m_{r t s}\right)+a+S I F S+ \\
T_{c t s}\left(m_{c t s}\right)+a
\end{array}\right] .
$$

4. $\bar{B}_{f 4}:$ no-collision $+R T S+C T S+$ (all MPDUs corrupted). When the RTS/CTS handshaking is successful, then the average lost time occurs due to RTS and CTS control frame transmission is

$$
\begin{gathered}
\bar{B}_{f 4}=P_{t r} \cdot P_{S} \cdot S_{r t s} \cdot S_{c t s} \cdot\left(1-S_{d}\left(m, n_{f}\right)\right)\left[D I F S+T_{r t s}\left(m_{r t s}\right)+\right. \\
\left.\quad a+S I F S+T_{c t s}\left(m_{c t s}\right)+a+S I F S\right] .
\end{gathered}
$$

\section{5. $\bar{B}_{f 5}$ : no-collision $+R T S+C T S+$ (some MPDUs corrupted).}

It models the waste time due to transmission of MPDUs frames that were corrupted due to noise and interference in spite of the RTS/CTS handshaking had been successful is given by

$$
\bar{B}_{f 5}=P_{s} \cdot P_{t r} \cdot S_{r t s} \cdot S_{c t s} \cdot\left\{\left(n_{f}-\overline{N_{f}}\right) \cdot\left(T_{d}\left(m_{d}\right)+S I F S+a\right)\right\}(35)
$$

5. $\bar{B}_{f 6}$ :no-collision $+R T S+C T S+\left(n_{f} M P D U s\right)+(A C K$ corrupted $)$. In this case does not matter if the MPDUS were corrupted or not because the ACK control frame is incapable to transmit this information to the target STA. Hence, the average time that the channel is busy transmitting unsuccessful ACK control frames must consider that $n_{f}$ MPDU are transmitted in an atomic cycle if the RTS/CTS handshaking was successful.

$$
\begin{aligned}
\bar{B}_{f 6}= & P_{s} \cdot P_{t r} \cdot S_{r t s} \cdot S_{c t s} \cdot\left(1-S_{a c k}\right) . \\
& \left\{D I F S+T_{r t s}\left(m_{r t s}\right)+a+S I F S+T_{c t s}\left(m_{c t s}\right)+a+S I F S\right. \\
& \left.+n_{f} \cdot\left(T_{d}\left(m_{d}\right)+S I F S+a\right)+\left[T_{a c k}\left(m_{a c k}\right)+a\right]\right\}
\end{aligned}
$$

The random backoff mechanism of CSMA/CA is designed to handle with collisions, i.e. the $\mathrm{CW}$ is doubled at each unsuccessful transmission in order to handle with the traffic load. Hence, the enhanced 802.11 MAC must reset the $\mathrm{CW}$ counter if any one of the MPDUs were transmitted with success. This is a logical issue because it is unnecessary to increase the $\mathrm{CW}$ when the failure of transmitting additional MPDUS occurred due to the radio channel environment and not due to the traffic load. Founded on this remark, we may use (1) and (2) in order to estimate $p$ and $\tau$.

\section{B. CONCATENATION (CONCT)}

Fig. $2 \mathrm{~b}$ depicts the time diagram of the concatenation protocol. The concatenation header $(\mathrm{CH})$ has a filed to indicate the number of frames concatenated. Given that the channel was successfully reserved by the RTS/CTS clearing technique, then the source STA transmits $n_{f}$ concatenated MPDUs. The ACK control frame has different fields to acknowledge each transmitted MPDU separately.

The CONCT protocol is analogous to the MFT-V1 scheme, except that now the frames are concatenated without SIFS between them. Hence, the goodput can be estimated by (25), where the mean number of MPDU frames transmitted in the cycle time is given by (24). Emphasizing again that an atomic cycle is successful if at least one data frame is transmitted with success, then the average cycle time is given by

$$
\bar{T}=\bar{B}_{s}+\bar{B}_{f 1}+\bar{B}_{f 2}+\bar{B}_{f 3}+B_{f 4}+B_{f 5}+B_{f 6}+\bar{I}
$$

The average busy time spent for a successful transmission is given by (compare with 29)

$$
\begin{aligned}
\bar{B}_{S}= & P_{s} \cdot P_{t r} \cdot S_{r t s} \cdot S_{c t s} \cdot S_{a c k} \\
& \left\{\left[D I F S+T_{r t s}\left(m_{r t s}\right)+a+S I F S+T_{c t s}\left(m_{c t s}\right)+a+S I F S\right]+\right. \\
& \left.\overline{N_{f}} \cdot\left(T_{d}\left(m_{d}\right)+a\right)+S I F S+T_{a c k}\left(m_{a c k}\right)+a\right\}
\end{aligned}
$$

The following time periods model the average waste time in which the channel is busy with unsuccessful frame transmissions:

1. $\bar{B}_{f 1}, \bar{B}_{f 2}$ and $\bar{B}_{f 3}$.

These busy times are given by (31-33) since these events are independent of the MAC protocol enhancement scheme used.

2. $\bar{B}_{f 4}:$ no-collision $+R T S+C T S+$ (all MPDUs corrupted).

It is given by (34) since the CONCT scheme does not change the RTS/CTS handshaking.

3. $\bar{B}_{f 5}$ : no-collision $+R T S+C T S+($ some MPDUs corrupted).

The waste busy time due to corrupted transmission of additional MPDUs when RTS/CT handshaking is successful is analogous to (35), except that for CONCT scheme the average number of corrupted MPDUs must multiply $\left(T_{d}\left(m_{d}\right)+a\right)$ and not $\left(T_{d}\left(m_{d}\right)+a+S I F S\right)$, as in (35).

$$
\bar{B}_{f 5} \approx P_{S} \cdot P_{t r} \cdot S_{r t s} \cdot S_{c t s} \cdot\left\{\left(n_{f}-\overline{N_{f}}\right) \cdot\left(T_{d}\left(m_{d}\right)+a\right)\right\}
$$

\section{4. $\bar{B}_{f 6}:$ no-collision $+R T S+C T S+\left(n_{f} M P D U s\right)+(A C K$ corrupted $)$.}

The waste time due to an unsuccessful transmission of an ACK control frame) is given by (40) since an atomic cycle consists of $n_{f}$ 
MPDUs if the RTS/CTS clearing technique is successful.

$$
\begin{aligned}
\bar{B}_{f 6}= & P_{t r} \cdot P_{S} \cdot S_{r t s} \cdot S_{c t s} \cdot\left(1-S_{a c k}\right)\left[D I F S+T_{r t s}\left(m_{r t s}\right)+\right. \\
& a+\operatorname{SIFS}+T_{c t s}\left(m_{c t s}\right)+a+\operatorname{SIFS}+\cdot n_{f} \cdot\left(T_{d}\left(m_{d}\right)+a\right)+ \\
& \left.\operatorname{SIFS}+T_{a c k}\left(m_{a c k}\right)+a\right] .
\end{aligned}
$$

The random backoff mechanism of CSMA-CA is designed to handle with collisions, i.e. the $\mathrm{CW}$ is doubled at each unsuccessful transmission. Therefore, as in MFT scheme the enhanced 802.11 MAC must reset the $\mathrm{CW}$ if any one of the data is transmitted with success. Hence, we can use (1) and (2) in order to estimate $p$ and $\tau$.

\section{SYSTEM PERFORMANCE}

The simulation results are obtained using an IEEE 802.11 joint MAC and PHY simulator that has been developed using $\mathrm{C}$ object oriented language:

- It implements an ad hoc IEEE 802.11a PHY layer.

- It implements the MAC state machine that fulfills the IEEE 802.11 DCF plain and enhanced BA and RTS/CTS schemes.

- The OFDM PHY layer is implemented assuming perfect synchronism. The PHY layer signal processing algorithms implements the maximum-likelihood hard decision detection for the PHY mode 1 to PHY mode 8.

- The convolutional hard-decision decoding is implemented using a semi-analytic approach as follows. The short-term average BER is estimated at a frame basis using on-line statistics collected at the demodulator output. Then the average BER is used in (16-17) to estimate the probability that the hard decision Viterbi decoding algorithm produces a decoding error.

- It is assumed the IEEE 802.11a PHY layer parameters [8, pp. 279]: slot time $\sigma=9 \mu S, S I F S=16 \mu s, D I F S=34 \mu s, C W_{\min }=16$, $C W_{\max }=1023, m=6$. The propagation delay $a$ is set $t o 1 \mu$.s.

- The correlated fading (tantamount for block fading) is generated using the Jakes' model with carrier frequency of $5.5 \mathrm{GHz}$ and velocity of $3 \mathrm{~km} / \mathrm{h}$.

- It is assumed a confidence interval of $98 \%$.

We show analytical and simulation results in Fig 3a (enhanced 802.11 MFT protocol using PHY mode 5), Fig. 3b (enhanced 802.11 MFT protocol using PHY mode 8), Fig 4a (enhanced 802.11 CONCT protocol using PHY mode 5) and Fig 4b (enhanced 802.11 CONCT protocol using PHY mode 8). These figures also have shown results for the standardized plain IEEE DCF 802.11 MAC protocol. In spite of the high complexities involved in modelling the IEEE 802.11 MAC protocols, we claim that our proposed fist order model propitiates a good agreement between analytical and simulation results for transmission of two and three frames.

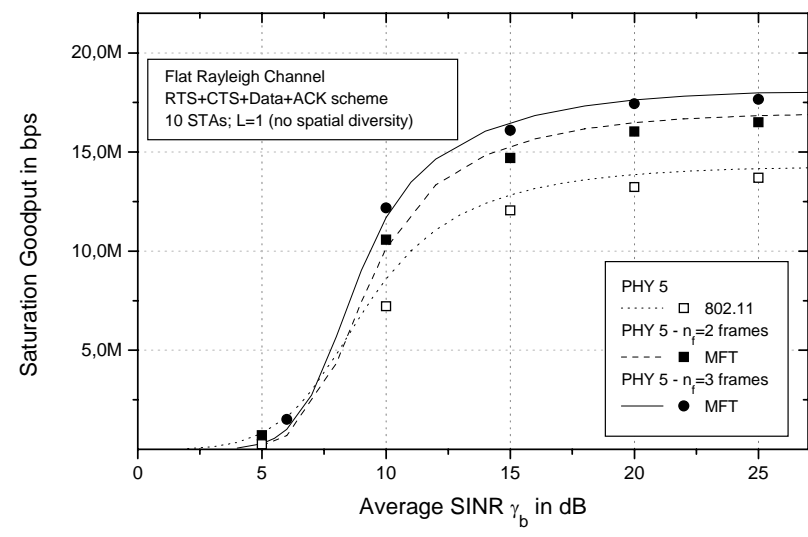

Fig. 3a - PHY mode 5 .

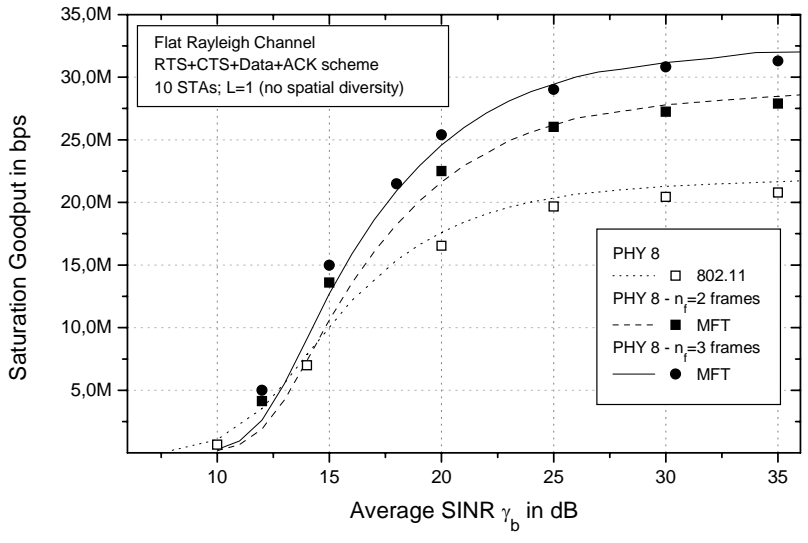

Fig. $3 b$ - PHY mode 8 .

Fig. 3 - Comparison between analytical (straight lines) and simulation (marks) results for MFT MAC protocol: $l_{p l}=1023$ octets, $n=10$ STAs.

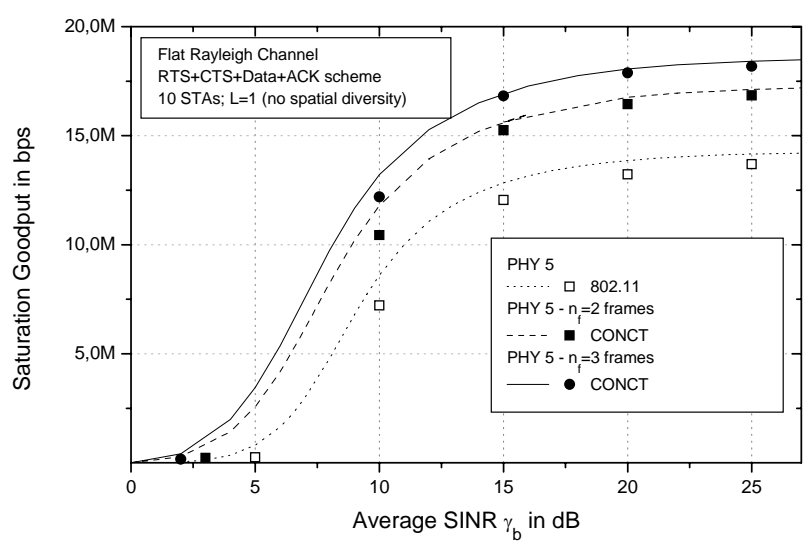

Fig. 4b - PHY mode 5.

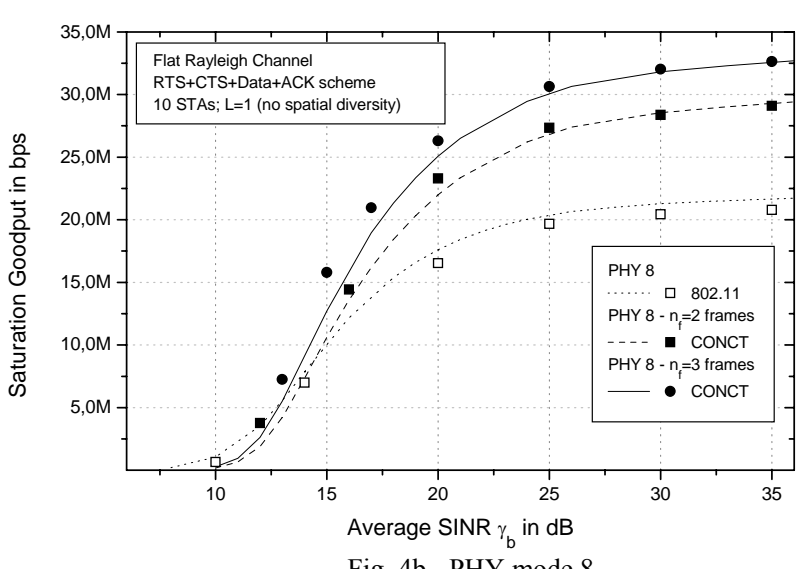

Fig. 4b - PHY mode 8 .

Fig. 4 - Comparison between analytical (straight lines) and simulation (marks) results for CONCT MAC protocol: $l_{p l}=1023$ octets, $n=10$ STAs.

The data shown in Tab I (PHY mode 5) and Tab. II (PHY mode 8) summarize the achievable net throughput for the different MAC schemes at high SINR. We can readily verify a considerable capacity improvement due to the MAC enhancements mechanisms. Both MFT and CONCT protocols transmit only one ACK frame at the end of the atomic cycle. However, the superior performance of the CONCT protocol is due to the lesser overhead since there is not SIFS between MPDUs frames.

Analysing the data shown at Figures 3 and 4 and Tables I and II, we can conclude that the gain due to MAC protocols enhancements are directly proportional to the number of 
frames and data rate. We observe that the greater these parameters are, then lesser are the effects of MAC protocol overheads, such as control frames, IFS, headers.

Tab. I - Goodput of different MAC protocols: PHY mode 5. $\mathrm{SINR}=25 \mathrm{~dB}$.

\begin{tabular}{|c|c|c|c|c|}
\hline \multirow{2}{*}{$\begin{array}{c}\text { Protoc } \\
\text { ol }\end{array}$} & \multicolumn{2}{|c|}{ 2 Frames } & \multicolumn{2}{c|}{ 3 Frames } \\
\cline { 2 - 5 } & Goodput & Gain & Goodput & Gain \\
\hline 802.11 & $14.29 \mathrm{Mbps}$ & ---- & $14.29 \mathrm{Mbps}$ & ---- \\
\hline MFT & $16.83 \mathrm{Mbps}$ & $17.77 \%$ & $17.98 \mathrm{Mbps}$ & $25.82 \%$ \\
\hline CONCT & $17.18 \mathrm{Mbps}$ & $20.22 \%$ & $18.48 \mathrm{Mbps}$ & $29.32 \%$ \\
\hline
\end{tabular}

Tab. II - Goodput of different MAC protocols: PHY mode 8.

\begin{tabular}{|c|c|c|c|c|}
\hline \multirow{2}{*}{ Protocol } & \multicolumn{2}{|c|}{ S Frames } & \multicolumn{2}{c|}{3 Frames } \\
\cline { 2 - 5 } & Goodput & Gain & $\begin{array}{c}\text { Goodput } \\
\text { Mbps }\end{array}$ & Gain \\
\hline 802.11 & $21.72 \mathrm{Mbps}$ & ---- & $21.72 \mathrm{Mbps}$ & ---- \\
\hline MFT & $28.60 \mathrm{Mbps}$ & $31.67 \%$ & $32.02 \mathrm{Mbps}$ & $47.42 \%$ \\
\hline CONCT & $29.42 \mathrm{Mbps}$ & $35.45 \%$ & $32.70 \mathrm{Mbps}$ & $50.55 \%$ \\
\hline
\end{tabular}

Fig. 5 shows the goodput versus the number of STAs in the system. These results are parameterised by the MAC protocols. It is assumed the PHY mode 8 , SINR of $36 \mathrm{~dB}$, $n_{f}=3$ frames and a MPDU payload of 1023 octets. We verify a good agreement between analytical and simulation results and a minor dependence between the goodput and channel load.

Fig. 6 shows the goodput versus the MPDU payload per frame given in bytes. These results are parameterised by the MAC protocols. It is assumed the PHY mode 8, SINR of 36 $d B, n_{f}=3$ frames a traffic load of 10 STAs. The goodput increases substantially with the MPDU length due to the reduction of MAC overhead effects (e.g. the various IFS, headers, control frames).

\section{CONCLUSIONS}

In this contribution we used our previous analytical results on IEEE 802.11a DCF MAC protocol [7] (summarized in sections II and III) in order to derive a first order theoretical MAC and PHY cross-layer model that allows estimating the net throughput (goodput) of IEEE 802.11n-based DCF MAC enhancements. These MAC improvements consist of modifications of the IEEE 802.11 distribution DCF MAC protocol in order to support concatenation and multiframe transmission techniques.

These improved MAC schemes can allow performance gains of $\sim 36 \%$ (with concatenation of two frames using PHY mode 8 ) and $\sim 50 \%$ (with concatenation of three frames using PHY mode 8) in relation to the plain 802.11 DCF MAC protocol (see Tab. I and II). The goodput supplied by these MAC enhancement schemes has a minor dependence with the channel load (see Fig. 5). However, their performance increases with data rate (compare Tab. I with Tab. II) and MPDU payload (see Fig. 6).

As future research activities, we have been extending the current analytical model to jointly analyze MAC and MIMO PHY layer enhancements for different spatial-timefrequency channel models.

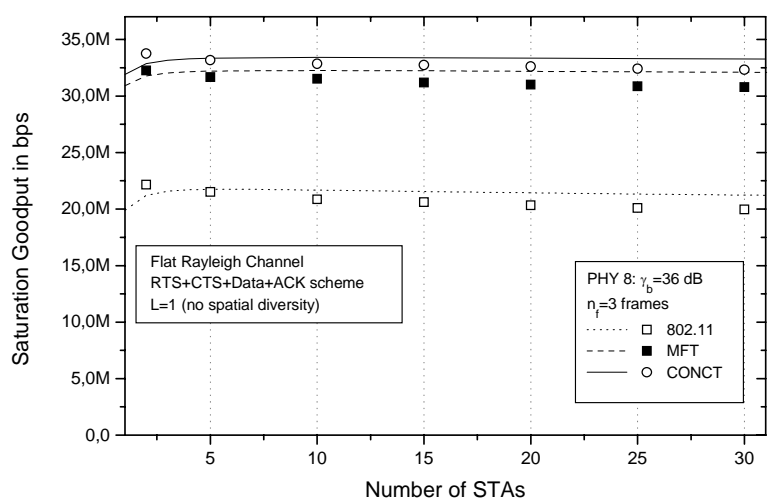

Fig. 5 - Comparison between analytical (straight lines) and simulation (marks) results for the net throughput versus the number of STAs.

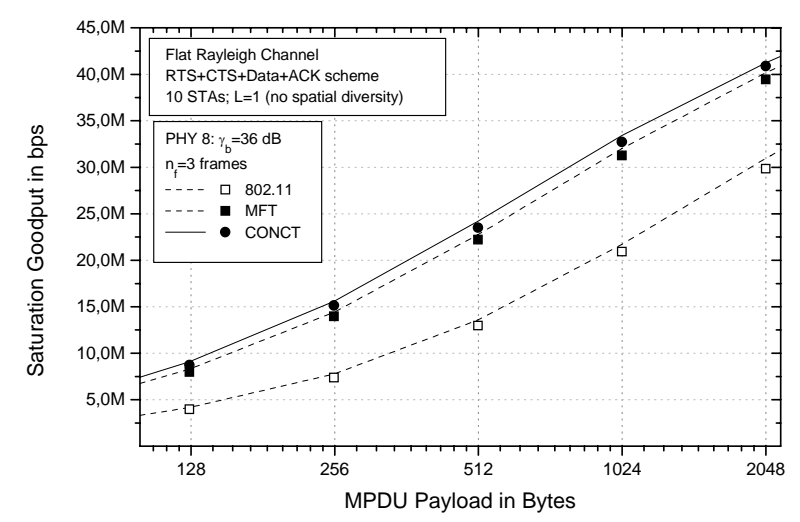

Fig. 6 - Comparison between analytical (straight lines) and simulation (marks) results for the net throughput versus the MPDU payload in bytes.

\section{REFERENCES}

[1] Y. Xiao and J. Rosdahl. "Throughput and delay limits for IEEE 802.11". IEEE Communications Letters, v. 6, no. 8, p. 355-357, Aug. 2002.

[2] Y. Xiao. "Packing mechanisms for the IEEE 802.11n wireless LANs". Globecom 2004, v. 5, p. 3275-3279, Nov. 2004.

[3] Y. Xiao. "IEEE 802.11n: enhancements for higher throughput in wireless LANs". IEEE Wireless Communications, v.12, n.6, p. 8291, Dec. 2005.

[4] C. Liu and A.P. Stephens. "An analytical model for infrastructure WLAN capacity with bidirectional frame aggregation". Wireless Communication and Networking Conference, v. 10, p. 4811-4817, June 2006.

[5] L. X. Cai et. al. "Capacity analysis of enhanced MAC in IEEE 802.11 ". First International Conference on Communications and Networking in China. p. 1-5, Oct. 2006.

[6] K-C Ting, H-H Lee, F. Lai. "Design and analysis of enhanced grouping DCF scheme for the MAC layer enhancement of 802.11n with ultra-high data rate". $4^{\text {th }}$ Symposium on Wireless Communication Systems (ISWCS 2007), p. 252-256, Oct. 2007.

[7] R. P. F. Hoefel. "A MAC and PHY cross-layer analytical model for the goodput and delay of IEEE 802.11a networks operating under basic access and RTS/CTS DCF schemes", Journal of Communications (JCM), v.1, n.6, p. 1-8, Set. 2006.

[8] S. Gast, 802.11 Wireless Networks. New York: O’Reilly, 2005.

[9] G. Bianchi "Performance Analysis of the IEEE 802.11 Distributed coordination function," IEEE Journal on Select Areas on Comm., v.18, no. 3, pp. 535-547, March 2000.

[10] M. B. Pursle and D. J. Taipale. "Error probabilities for spreadspectrum packet radio with convolutional codes and Viterbi decoding”, IEEE Trans. Commun., vol. 35, n. 1, p. 1-12, Jan. 1987.

[11] R. P. F. Hoefel, C. de Almeida. "The performance of CDMA/PRMA for Nakagami-m frequency selective fading channel", Electronics Letters, vol. 35, no. 1, 1999, pp 28-29.

[12] L. Yang and Hanzo, L. "A recursive algorithm for the error probability evaluation of M-QAM," IEEE Comm. Letters, vol. 4, pp. 304-306, 2000. 